\title{
Histomorphometric classification of postmenopausal osteoporosis: implications for the management of osteoporosis
}

\author{
M T A Rehman, J A Hoyland, J Denton, A J Freemont
}

\begin{abstract}
Aims-To define and group static and dynamic iliac crest histomorphometric parameters in women with established osteoporosis.

Methods-Iliac crest biopsy specimens from 146 white women were sectioned undecalcified and examined using image analysis.

Results-Five distinct groups were defined on the basis of histomorphometric changes in cell function: group 1, decreased osteoblastic and osteoclastic activity; group 2 , decreased osteoblastic and increased osteoclastic activity; group 3 , increased osteoblastic and osteoclastic activity; group 4 , no bone surface cell activity; and group 5, apparently normal osteoblastic and osteoclastic activity.

Conclusions-Five distinct subgroups of patients with postmenopausal osteoporosis can be defined based on changes in bone cell function. Defining cellular dysfunction in this way may be important for tailoring treatment regimens to the needs of individual patients.

(F Clin Pathol 1995;48:229-235)
\end{abstract}

Keywords: Histomorphometric parameters, osteoporosis

Postmenopausal osteoporosis is the commonest type of osteoporosis in clinical practice. Despite increased awareness of the condition and its importance, most women still present with the established disorder, resulting in all the clinical problems of managing patients with a symptomatic disorder but without a satisfactory treatment regimen. Patients are currently grouped on the basis of clinical characteristics. Response to treatment is unpredictable perhaps because current treatment regimens are unable to normalise the microstructural changes associated with osteoporosis. We hypothesise that the failure of single treatment regimens might also be because of the heterogeneous nature of the cellular changes in this group.

The clinical syndrome of osteoporosis is a common manifestation of a variety of disorders. At the cellular level, the syndrome can be broadly regarded as an imbalance or uncoupling of osteoblastic bone deposition and osteoclastic bone resorption. ${ }^{1}$ Theoretically, this imbalance may take several forms ranging from an increase in both osteoclasis and osteoblastic activity, with a greater increase in the former, ${ }^{2}$ to a decrease in both, with a greater reduction in osteoblastic activity. ${ }^{34}$

Studies of postmenopausal osteoporosis suggest that there is heterogeneity within this group. Some studies have shown that "uncoupling of bone" is caused by increased bone resorption. ${ }^{5}$ This may lead to perforation of bone trabeculae, occasionally with an increase in bone fragility greater than that expected from the amount of bone lost. ${ }^{34}$ Other studies suggest that the predominant abnormality is the failure of osteoblasts to fill resorbed spaces. ${ }^{67}$ There is no overall consensus concerning the nature of bone remodelling changes in postmenopausal osteoporosis. Whilst most studies on subjects with idiopathic osteoporosis have demonstrated low bone turnover, ${ }^{3-11}$ Recker et al ${ }^{11}$ reported trabecular microarchitectural changes associated with normal bone remodelling and Wands et $a l^{12}$ demonstrated that women with "vertebral crush syndrome" continue to have an inappropriately prolonged phase of increased bone remodelling. Generally, the same principles apply to cortical and trabecular bone loss. ${ }^{1314}$ There is no consensus as to the overall relation between clinically defined patient groups and the changes in bone cell function and matrix that typify the syndrome.

This perplexing diversity of opinion may have arisen because the cellular changes are heterogeneous. Recognition of specific parameters of bone turnover and the nature of the predominant cellular abnormalities in an individual or group of patients may be a more useful way to define osteoporosis, permitting selection of optimum therapy to improve or at least to reduce further deterioration of the biomechanical integrity of the skeleton. To test this hypothesis, we have used histomorphometry to obtain data on bone cell activity and function in 146 postmenopausal women with established osteoporosis.

\section{Methods}

All patients were recruited from either Hope Hospital, Salford, or the Manchester Royal Infirmary, Manchester. Women were regarded as postmenopausal if they had been amenorrhoeic for more than 12 months. Oestradiol and gonadotrophin concentrations were measured to confirm menopausal status. Diagnosis of established osteoporosis was determined radiologically by the presence of at least one non-traumatic vertebral crush 
fracture or a lumbar spine (L2-L4) bone mineral density (BMD) less than 2 SD below peak bone mass (assessed by DXA). All secondary causes of osteoporosis were excluded following appropriate investigations: determination of parathyroid hormone, serum calcium, phosphate, and alkaline phosphatase concentrations, thyroid function tests, and immunoglobulin electrophoresis.

To distinguish between postmenopausal and senile osteoporosis, an arbitrary upper age limit was set as recommended by Riggs and Melton. ${ }^{15}$ Only women between 45 and 75 years of age were studied and only those patients with an adequate amount of both cortical and trabecular tissue in the iliac crest bone biopsy specimen were included.

All patients were given oral dimethyl-chlortetracycline (10-15 mg/kg body weight) in divided doses over 12 hours 15-18 days before bone biopsy. This dose schedule was repeated after an interval of 10 days. Early morning urine specimens were collected before each tetracycline dose schedule and a day later to confirm compliance and adequate absorption of tetracycline. Biopsy was performed within four to seven days of the last tetracycline dose. A full blood count and coagulation profile were performed before biopsy to exclude any bleeding disorders.

Bone biopsy was performed under local anaesthesia. Full thickness transiliac bone biopsy specimens were taken, $2 \mathrm{~cm}$ below and behind the anterior superior iliac spine, ${ }^{16}$ using a Lalor trephine biopsy needle. None of these patients had previously undergone biopsy at that site. Haemostasis was routinely secured by a couple of superficial stitches and a pressure bandage was applied for 12 hours.

Biopsy specimens were fixed in absolute alcohol and processed three times in LR white resin monomer (London Resin Co.), the last two taking place under reduced pressure. Resin polymerisation was carried out overnight at $60^{\circ} \mathrm{C}$. Twenty seven $5 \mu \mathrm{m}$ step serial sections were cut through each block with a Tungsten tipped knife on a LKB powered microtome. Groups of three sections were stained with toluidine blue. $(\mathrm{pH} 4 \cdot 2)$, or using the modified Giemsa or Von Kossa techniques. For fluorescence microscopy, $20 \mu \mathrm{m}$ unstained sections were cut at different levels throughout the block.

A semiautomated VIDS II image analyser was used to derive area, length, number, and two point analyses on each of nine toluidine blue stained sections by a single experienced operator. The mineral apposition rate (MAR), however, was measured using an eyepiece graticule in the fluorescence microscope to perform two point analysis at four regularly spaced positions along each front delineated by double fluorescent label. ${ }^{17}$ Microscopic magnification varied from $\times 40$ for area measurements to $\times 160$ for two point analyses and cell counting. Where data were expressed as ratios of similar parameters, conversion of measured two-dimensional to derived threedimensional numerics required no change in the ratio, as defined by the theorem of DeLesse, ${ }^{18}$ but where spherical geometry was involved, the stereologic constant $\pi / 4$ was used to convert two-dimensional measurements into three-dimensional measurements. ${ }^{19}$

When tetracycline labelled sections were used, the length of mineralising surface was divided into two components-those with two and those with only one label. The latter represents regions of the bone surface that either finished or started mineralising in the interval between administration of the two labels. To define certain parameters of bone formation more accurately, a corrected value of the length of the mineralising surface was constructed, taking account of single labelling, using the standard method of defining the true active mineralising surface as the double labelled plus half the single labelled surfaces (dLs+1/2sLs).

Toluidine blue stained sections were used to identify the osteoid surface, defined as an unmineralised surface at least $3 \mu \mathrm{m}$ thick. Osteoid width (O.Wi) was then measured at four equidistant sites on each osteoid seam. All measurements were based on the mean of 20 separate fields using the standard constant for section obliquity $(1 \cdot 2)$.

Parameters were measured using standard techniques ${ }^{17}{ }^{19-21}$ and defined according to the terminology proposed by Parfitt et al. ${ }^{22}$ The static and dynamic parameters obtained by measurement or calculation are presented in table 1 .

\section{Results}

All histomorphometric parameters are presented as a mean $z$-score. A $z$-score for an individual value is the number of standard deviations by which the value differs from the mean of normal age and sex matched controls for the local population. ${ }^{23}$ Using the $\mathrm{z}$-score, data from different age groups with different normal values can be pooled. The mean $z$ score can be calculated for a group as well as an individual. A difference of more than $3 \mathrm{z}$ score units (that is, $3 \mathrm{SD}$ above or below normal) between the patient groups and controls is regarded as abnormal; any parameter with a $z$-score between 2 and 3 is classified as moderately abnormal and is only marginally or slightly abnormal if the $\mathrm{z}$-score is between 1 and 2 . The parameters are regarded as normal if the $\mathrm{z}$-score is less than 1.

Patients could be divided into five distinct subgroups based on the pattern of overall changes in certain histomorphometric parameters (z-score, factor and cluster analysis). Osteoblast parameters included osteoblast surface, osteoid surface (dLs $+1 / 2 \mathrm{sLs}$ ) mineralisation lag time, bone formation rate, and osteoclast parameters included eroded surface, osteoclast number, osteoclast surface, and erosion period (figure). The numeric data for all the patients are presented in tables 2 to 5

Group 1 comprised $85(58 \%)$ patients. Trabecular bone in this group was charac- 
Table 1 Histomorphometric parameters: methods of measurement or calculation

\begin{tabular}{|c|c|c|}
\hline Parameters & Abbreviation & Methods \\
\hline $\begin{array}{l}\text { Activation frequency } \\
\text { Adjusted appositional rate`} \\
\text { Bone formation rate } \\
\text { Bone surface } \\
\text { Bone volume } \\
\text { Cortical thickness } \\
\text { Cortical volume } \\
\text { Erosion depth } \\
\text { Active erosion period } \\
\text { Erosion rate } \\
\text { Eroded surface (absolute) } \\
\text { Active eroded surface } \\
\text { Eroded surface 4(relative) } \\
\text { Formation period } \\
\text { Label interval } \\
\quad \text { double label surface } \\
\text { inter label distance } \\
\text { single label surface } \\
\text { total label surface } \\
\text { Mineralisation apposition rate (cortical) } \\
\text { Mineralisation apposition rate (trabecular) } \\
\text { Mineralisation lag time } \\
\text { Mineralising surface (corrected) } \\
\text { Mineralising surface (double) } \\
\text { Mineralising surface total (absolute) } \\
\text { Mineralising surface absolute (bone }\end{array}$ & $\begin{array}{l}\text { Ac.f (days) } \\
\text { Aj.AR }(\mu \mathrm{m} / \text { day }) \\
\text { BFR/BV (\% year) } \\
\text { BS }(\mu \mathrm{m}) \\
\text { BV }(\%) \\
\text { C.Th }(\mu \mathrm{m}) \\
\text { CV }\left(\mathrm{mm}^{3}\right) \\
\text { E.De }(\mu \mathrm{m}) \\
\text { a.EP }(\text { days }) \\
\text { ER (mm/year) } \\
\text { ES }(\mu \mathrm{m}) \\
\text { a.ES }(\mu \mathrm{m}) \\
\text { ES/BS }(\%) \\
\text { FP (days) } \\
\text { LP (days) } \\
\text { dLs }(\mu \mathrm{m}) \\
\text { iLs }(\mu \mathrm{m}) \\
\text { sLs }(\mu \mathrm{m}) \\
\text { tLs }(\mu \mathrm{m}) \\
\text { MARc }(\mu \mathrm{m} / \text { day) } \\
\text { MARt }((\mu \mathrm{m} / \text { day) } \\
\text { Mlt }(\text { days }) \\
\text { MS.c }(\mu \mathrm{m}) \\
\text { Msd }(\%) \\
\text { MSt }(\mu \mathrm{m})\end{array}$ & $\begin{array}{l}1 /(\mathrm{EP}+\mathrm{FP}+\mathrm{QP}) \\
(\mathrm{MS} / \mathrm{OS}) \times \mathrm{MAR} \\
(\mathrm{MAR} \times \mathrm{MS} \times \mathrm{BS} / \mathrm{BV}) 100 \\
\mathrm{LM} \\
\mathrm{AM}(\mathrm{BV} / \mathrm{TV}) \times 100 \\
2 \mathrm{PM} \times \pi / 4 \\
\mathrm{AM}(\mathrm{CV} / \mathrm{TV}) \times 100 \\
2 \mathrm{PM} \\
(\text { a.ES} / \mathrm{OS}) \times \mathrm{FP} \\
\mathrm{E} . \mathrm{De} / \mathrm{a} . \mathrm{EP} \times 1 / 1000 \times 365 \\
\mathrm{LM} \\
\mathrm{LM} \\
(\mathrm{ES} / \mathrm{BS}) \times 100 \\
\text { W.Th/Aj.AR } \\
\text { time between labels } \\
\mathrm{LM} \\
\mathrm{LM} \\
\text { sLs+dLs } \\
2 \mathrm{PM} \\
\text { iLd/LP } \\
\text { iLd/LP } \\
\text { O.Th/Aj.AR } \\
\text { dLs+1/2Ls } \\
\text { dLs/tLs } \\
\text { tLs }\end{array}$ \\
\hline $\begin{array}{l}\text { Mineralising surface (osteoid referent) } \\
\text { Number of osteoclasts (cortical) } \\
\text { Number of osteoclasts (trabecular) } \\
\text { Number of osteoclasts (subcortical) } \\
\text { Osteoblast surface } \\
\text { Osteoclast surface } \\
\text { Osteoid surface (absolute) } \\
\text { Osteoid surface (relative) } \\
\text { Osteoid thickness } \\
\text { Osteoid width } \\
\text { Osteoid volume } \\
\text { Quiescent period } \\
\text { Quiescent surface` } \\
\text { Trabecular number } \\
\text { Trabecular separation } \\
\text { Trabecular thickness } \\
\text { Tissue volume } \\
\text { Wall thickness } \\
\text { Wall thickness (cortical) } \\
\text { Wall width }\end{array}$ & $\begin{array}{l}\text { MS/BS }(\%) \\
\text { MS/OS }(\%) \\
\text { N.Oc.c }\left(/ \mathrm{mm}^{2}\right) \\
\text { N.Oc.t }\left(/ \mathrm{mm}^{2}\right) \\
\text { N.Oc.s }\left(/ \mathrm{mm}^{2}\right) \\
\text { Ob.S }(\%) \\
\text { Oc.S }(\%) \\
\text { OS }(\mu \mathrm{m}) \\
\text { OS/BS }(\%) \\
\text { O.Th }(\mu \mathrm{m}) \\
\text { O.Wi }(\mu \mathrm{m}) \\
\text { OV }(\%) \\
\text { QP (days) } \\
\text { QS (um) } \\
\text { TN }\left(/ \mathrm{mm}^{2}\right) \\
\text { TS }(\mu \mathrm{m}) \\
\text { T.Th }(\mu \mathrm{m}) \\
\text { TV }\left(\mathrm{mm}^{2}\right) \\
\text { W.Th }(\mu \mathrm{m}) \\
\text { W.Th.c }(\mu \mathrm{m}) \\
\text { W.Wi }(\mu \mathrm{m})\end{array}$ & $\begin{array}{l}\text { MS/BS (dLs+1/2sLs) } \\
\text { MS/OS (sLs+dLs) } \\
10^{-2} \text { (NOc.c/TV.c) } \\
10^{-2} \text { NOc.t/TV.t) } \\
10^{-2} \text { NOc.s/LM } \\
\text { Ob.S/BS } \\
\text { Oc.S/BS } \\
\text { LM } \\
(\text { OS/BS }) \times 100 \\
\text { OWi } \times \pi / 4 \\
2 \mathrm{PM} \\
\text { OV/BV } \\
(\mathrm{QS}+\mathrm{OS}) \mathrm{FP} \\
\text { BS-(ES+OS) } \\
\text { numbers/area } \\
2 \mathrm{PM} \\
2 \mathrm{PM} \\
\text { AM } \\
2 \mathrm{PM} \times \pi / 4 \\
2 \mathrm{PM} \times \pi / 4 \\
2 \mathrm{PM}\end{array}$ \\
\hline
\end{tabular}

*Calculated parameters; LM, length measurement; $\mathrm{AM}$, area measurement;

2PM, two point measurement.
Table 1 abbreviations:

$M A R=$ mineral apposition rate

dLs = double labelled surface

sLs = single labelled surface

Tb.N $=$ trabecular number
Tb. Th $=$ trabecular thickness

Tb. $T h=$ trabecular thick
W. $T h=$ wall thickness

TBV = trabecular bone volume

$\mathrm{OS} / \mathrm{BS}=$ osteoid surface

MARt $=$ mineral apposition rate in trabecular bone

$\mathrm{BFR} / \mathrm{BS}=$ bone formation rate at bone surface

$\mathrm{BER} / \mathrm{BV}=$ bone formation rate to bone volume

Aj.AR = adjusted apposition rate

Mlt $=$ mineralisation lag time

$\mathrm{FP}=$ formation period

Ac. $f=$ activation frequency

$\mathrm{ES} / \mathrm{BS}=$ eroded surfaces

$\mathrm{OcS} / \mathrm{BS}=$ osteoclast surface

EP $=$ erosion period

Ct. Th $=$ cortical thickness

$\mathrm{CV}=$ cortical volume

W. The $=$ cortical wall thickness

MAR c = mineral apposition rate in cortical bone

E.De $=$ erosion depth

a.Ep $=$ active erosion period

$\mathrm{ER}=$ erosion rate

$\mathrm{ES}=$ eroded surface (absolute)

a.ES = active eroded surface

ES/BS = eroded surface (relative)

LP = label interval

iLs $=$ inter label distance

$\mathrm{Ls}=$ total label surface

MS.c $=$ mineralising surface $($ corrected $)$

MSd = mineralising surface (double)

$\mathrm{MSt}=$ mineralising surface total (absolute)

MS/BS = mineralising surface absolute (bone referent)

MS/OS = mineralising surface (osteoid referent)

NOc.c $=$ number of osteoclasts (cortical)

NOc.t $=$ number of osteoclasts (trabecular)

NOc.s $=$ number of osteoclasts (subcortical)

$\mathrm{Ob} . \mathrm{S}=$ osteoblast surface

Oc. $S=$ osteoclast surface

OS = osteoid surface (absolute)

OS/BS = osteoid surface (relative)

O.Th $=$ osteoid thickness

$\mathrm{O} . \mathrm{Wi}=$ osteoid width

OV = osteoid volume

$\mathrm{QP}=$ quiescent period

$\mathrm{QS}=$ quiescent surface

$\mathrm{TN}=$ trabecular number

TS = trabecular separation

TV $=$ tissue volume terised by a low bone volume, reduced wall thickness, and a decreased appositional rate secondary to diminished osteoblast cell number and activity. These parameters progressively decreased with increasing age. The osteoid surface, volume and thickness as well as mineralising surface (corrected) (measured as double label surface $+1 / 2$ single label surface) were normal. The mineralising surface, whether measured as double label surface plus single label surface (more than $-2 \cdot 0$ ) or as the sum of double and total label surfaces (more than-3.5), was moderately to notably reduced. Trabecular thickness and number decreased with increasing age in this group and hence trabecular separation increased with age $(+1.0$ at $45-50$ years to more than +3.0 at 50 years or over). Trabecular structure was largely fragmented-that is, trabeculae were perforated, and appeared discontinuous in sections (82\%); however, a

Table 2 The characteristics of patients in the different histomorphometric groups

\begin{tabular}{lll}
\hline Group & Total No. (\%) & Age (SD) in years \\
\hline 1 & $76(56)$ & $59(9)$ \\
2 & $34(25)$ & $67(8)$ \\
3 & $15(11)$ & $56(10)$ \\
4 & $4(3)$ & $48(4)$ \\
5 & $7(5)$ & $51(8)$ \\
\hline
\end{tabular}

small proportion was either normal (4\%) or of the filigree type (14\%)-that is, the trabeculae were continuous but appeared generally thin and frail in tissue sections. Both the fragmented and filigree types of trabeculae are abnormal and result in reduced bone strength. Recognition of the abnormal pattern of trabeculae has important therapeutic relevance. The structure of trabeculae is of prime clinical importance; in the fragmented type the trabecular scaffold is discontinuous and, therefore, is theoretically less amenable to bone forming therapy and disproportionately increases the risk of further fracture. On the whole, patients in group 1 had a noticeably reduced rate of bone formation $(-1.9$ to $-3 \cdot 8)$, as expressed by the ratio of bone formation to bone volume (BFR/BV), and although the bone formation period was normal in younger patients (z-score +0.1 for those aged from 45 to 50 years), it decreased progressively with age $(-2 \cdot 1$ for those aged from 60 to 65 years). Mineralising surfaces were noticeably reduced but the mineralisation lag time was only marginally increased $(+0.4$ to $+1 \cdot 1)$, suggesting that the predominant abnormality is impairment of the initiation of bone formation. The activation frequency was also reduced.

Analysis of cortical bone revealed very reduced wall thickness and a moderate 
Table 3 Static histomorphometric parameters in trabecular bone

\begin{tabular}{|c|c|c|c|c|c|c|c|c|c|c|c|c|c|c|c|c|c|}
\hline Group & $\begin{array}{l}\text { Age } \\
\text { (years) }\end{array}$ & No. & $T B V$ & $W . T h$ & $O S / B S$ & $O V$ & $O . T h$ & Ob.S & $M S$ & $M S$ & $M S d$ & $E S$ & $O c N$ & $O c S$ & $T b T h$ & $T N$ & $T S$ \\
\hline 1 & $\begin{array}{l}45-50 \\
50-55 \\
55-60 \\
60-65 \\
65-75\end{array}$ & $\begin{array}{r}21 \\
33 \\
17 \\
4 \\
1\end{array}$ & $\begin{array}{l}-1.6 \\
-2.9 \\
-3.1 \\
-2.8\end{array}$ & $\begin{array}{l}-1.4 \\
-2.5 \\
-3.5 \\
-3.3\end{array}$ & $\begin{array}{l}-0.2 \\
-0.5 \\
-0.4 \\
-0.3\end{array}$ & $\begin{array}{l}-1.2 \\
-1.2 \\
-0.9 \\
-0.9\end{array}$ & $\begin{array}{l}-0.7 \\
-0.6 \\
-0.6 \\
-0.5\end{array}$ & $\begin{array}{l}-1.2 \\
-1.2 \\
-0.9 \\
-0.9\end{array}$ & $\begin{array}{l}-0.4 \\
-0.4 \\
-0.9 \\
-1.0\end{array}$ & $\begin{array}{l}-2 \cdot 6 \\
-2 \cdot 4 \\
-2.5 \\
-2.3\end{array}$ & $\begin{array}{l}-4 \cdot 1 \\
-3 \cdot 7 \\
-3 \cdot 1 \\
-3.0\end{array}$ & $\begin{array}{l}-0.1 \\
+0.3 \\
+0.0 \\
+0.1\end{array}$ & $\begin{array}{r}-0.2 \\
+0.3 \\
+0.1 \\
0.0\end{array}$ & $\begin{array}{l}+0.3 \\
-0.5 \\
-0.4 \\
-0.4\end{array}$ & $\begin{array}{l}-0.2 \\
-0.4 \\
-0.8 \\
-0.8\end{array}$ & $\begin{array}{l}-0.7 \\
-1.9 \\
-1.9 \\
-1.7\end{array}$ & $\begin{array}{l}+1 \cdot 0 \\
+3 \cdot 3 \\
+4 \cdot 1 \\
+3 \cdot 1\end{array}$ \\
\hline 2 & $\begin{array}{l}45-50 \\
50-55 \\
55-60 \\
60-65 \\
65-70 \\
70-75\end{array}$ & $\begin{array}{r}1 \\
3 \\
4 \\
7 \\
8 \\
11\end{array}$ & $\begin{array}{l}-1 \cdot 7 \\
-2 \cdot 4 \\
-3 \cdot 6 \\
-3 \cdot 8 \\
-3 \cdot 7\end{array}$ & $\begin{array}{l}-2 \cdot 3 \\
-2 \cdot 8 \\
-2 \cdot 8 \\
-2 \cdot 5 \\
-2 \cdot 4\end{array}$ & $\begin{array}{l}+0.1 \\
-0.3 \\
+0.0 \\
-0.2 \\
-0.2\end{array}$ & $\begin{array}{l}-1.4 \\
-1.6 \\
-0.7 \\
-0.9 \\
-1.0\end{array}$ & $\begin{array}{l}-0.6 \\
-0.8 \\
-0.5 \\
-0.9 \\
-0.7\end{array}$ & $\begin{array}{l}-1 \cdot 7 \\
-2 \cdot 2 \\
-2 \cdot 1 \\
-1.9 \\
-2.0\end{array}$ & $\begin{array}{l}-1.1 \\
-1.5 \\
-1.9 \\
-1.3 \\
-1.6\end{array}$ & $\begin{array}{l}-1.5 \\
-1.7 \\
-1.8 \\
-1.9 \\
-1.8\end{array}$ & $\begin{array}{l}-3.6 \\
-3.0 \\
-2.9 \\
-3.1 \\
-2.4\end{array}$ & $\begin{array}{l}+2 \cdot 7 \\
+3 \cdot 2 \\
+3 \cdot 1 \\
+3 \cdot 0 \\
+3 \cdot 4\end{array}$ & $\begin{array}{l}+2.5 \\
+2.0 \\
+2.4 \\
+3.3 \\
+3.4\end{array}$ & $\begin{array}{l}+1.4 \\
+2.3 \\
+2.1 \\
+1.6 \\
+1.8\end{array}$ & $\begin{array}{l}-0.4 \\
-1.0 \\
-1.3 \\
-1.1 \\
-1.2\end{array}$ & $\begin{array}{l}-0.6 \\
-0.8 \\
-1.3 \\
-1.1 \\
-1.0\end{array}$ & $\begin{array}{l}+1 \cdot 1 \\
+3 \cdot 4 \\
+3 \cdot 7 \\
+3 \cdot 6 \\
+4 \cdot 0\end{array}$ \\
\hline 3 & $\begin{array}{l}45-55 \\
55-60 \\
65-75\end{array}$ & $\begin{array}{l}5 \\
5 \\
5\end{array}$ & $\begin{array}{l}-1.1 \\
-1.8 \\
-1.9\end{array}$ & $\begin{array}{l}+0.0 \\
+0.3 \\
-0.6\end{array}$ & $\begin{array}{l}+1 \cdot 0 \\
+1 \cdot 3 \\
+1 \cdot 1\end{array}$ & $\begin{array}{l}+0.7 \\
+0.2 \\
+0.0\end{array}$ & $\begin{array}{l}-0.2 \\
-0.2 \\
+0.0\end{array}$ & $\begin{array}{l}+0.7 \\
+0.8 \\
+0.9\end{array}$ & $\begin{array}{l}+0.1 \\
-0.1 \\
+0.4\end{array}$ & $\begin{array}{l}-0.4 \\
-0.4 \\
-0.5\end{array}$ & $\begin{array}{l}-0.4 \\
-0.3 \\
-0.6\end{array}$ & $\begin{array}{l}+1 \cdot 5 \\
+1 \cdot 1 \\
+1 \cdot 3\end{array}$ & $\begin{array}{l}+0.9 \\
+1.4 \\
+2.9\end{array}$ & $\begin{array}{l}+1.3 \\
+1.3 \\
+1.4\end{array}$ & $\begin{array}{l}-0.7 \\
-1.6 \\
-1.5\end{array}$ & $\begin{array}{l}+0.0 \\
-0.7 \\
-0.3\end{array}$ & $\begin{array}{l}+1 \cdot 3 \\
+2 \cdot 6 \\
+2.9\end{array}$ \\
\hline 4 & $45-50$ & 4 & $-2 \cdot 7$ & $-1 \cdot 6$ & $-3 \cdot 3$ & $-3 \cdot 8$ & $-3 \cdot 2$ & -0.7 & $+0 \cdot 1$ & $-1 \cdot 2$ & $-0 \cdot 2$ & $-1 \cdot 8$ & $-2 \cdot 2$ & -1.5 & -1.5 & +0.0 & $+1 \cdot 4$ \\
\hline 5 & $55-60$ & 3 & $-0 \cdot 1$ & +0.0 & $+0 \cdot 2$ & $+0 \cdot 1$ & $-0 \cdot 1$ & +0.0 & -0.4 & -0.3 & +0.2 & $+0 \cdot 2$ & +0.1 & $+0 \cdot 2$ & +0.3 & -0.1 & +0.0 \\
\hline
\end{tabular}

Units $=\mathrm{z}$-score, above $(+)$ or below $(-)$ the mean.
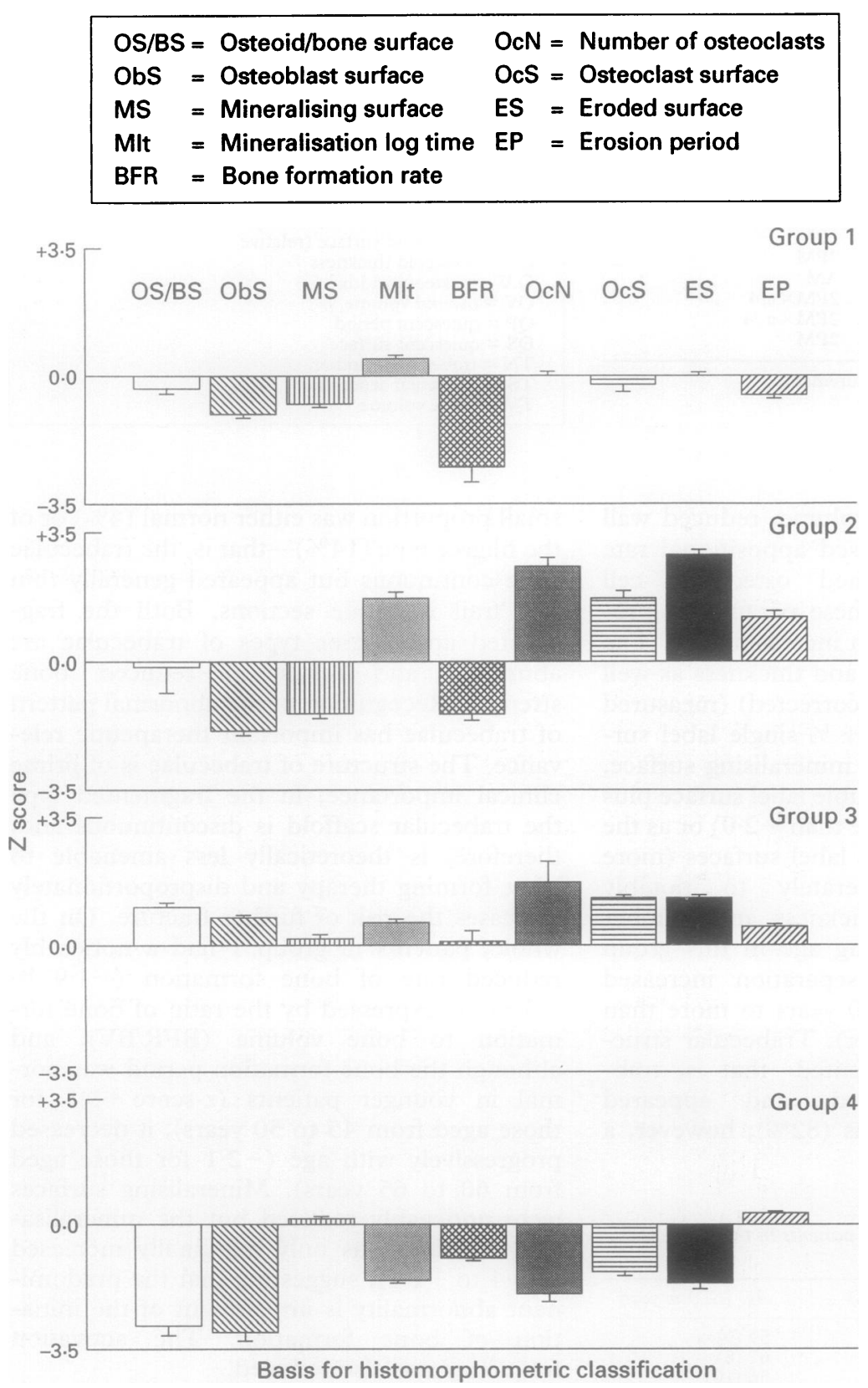

Patterns of significant histomorphometric parameters defining osteoporotic subgroups. increase in eroded surfaces, but the numbers of osteoclasts, and cortical thickness, cortical volume, and osteoid surfaces were normal. Although the number of osteoclasts in the subcortex was moderately elevated, the erosion rate and the erosion period were within the normal range. It was technically difficult to assess the rate of activation frequency in the cortex.

Group 2 comprised $32(22 \%)$ patients. These patients had reduced trabecular bone volume and wall thickness, as in patients in group 1, but the number of osteoblasts was moderately to noticeably reduced $(-1 \cdot 7$ to $-2 \cdot 2)$. The rate of bone formation $(\mathrm{BFR} / \mathrm{BV})$ was reduced but less so than in group 1 patients and the formation period was within the normal range. In contrast to group 1, however, group 2 patients had a mildly to moderately prolonged mineralisation lag time $(+1 \cdot 3$ to $+2 \cdot 1)$. The striking feature of trabecular bone in group 2 patients was the presence of a noticeable increase in the number of osteoclasts $(+2.0$ to +3.4$)$ associated with a similar increase in the number of eroded surfaces $(+2 \cdot 7$ to $+3 \cdot 4)$, but the erosion cavities were relatively shallow $(-1 \cdot 0$ to $-1 \cdot 2)$. Although the erosion rate was marginally decreased $(-0.6$ to $-1 \cdot 2)$, overall resorption was increased because the erosion period was also marginally longer $(+0.9$ to $+1 \cdot 8)$. On the whole, the rate of bone remodelling, as assessed by the activation frequency, was near the lower limit of the normal range. The trabecular pattern in most of the cases was fragmented and was of the filigree type in the remainder.

Cortical bone thickness was slightly reduced in group 2 patients when compared with those in group 1 and was associated with a mild to moderate increase in the number of osteoclasts both in the cortex and subcortex. There was a moderate increase in the number of eroded surfaces but the rate of erosion and the erosion period were normal, except in patients aged between 70 and 75 years, where they were marginally increased. 
Table 4 Dynamic histomorphometric parameters in trabecular bone

\begin{tabular}{|c|c|c|c|c|c|c|c|c|c|c|c|}
\hline Group & $\begin{array}{l}\text { Age } \\
\text { (years) }\end{array}$ & No. & $M A R t$ & $A j . A R$ & $M l t$ & $F P$ & $B F R / B V$ & E.De & $E P$ & $E R$ & Ac.f \\
\hline 1 & $\begin{array}{l}45-50 \\
50-55 \\
55-60 \\
60-65 \\
65-75\end{array}$ & $\begin{array}{r}21 \\
33 \\
17 \\
4 \\
1\end{array}$ & $\begin{array}{l}-1 \cdot 9 \\
-2 \cdot 1 \\
-2 \cdot 1 \\
-2 \cdot 4\end{array}$ & $\begin{array}{l}+1.4 \\
-1.6 \\
-1.6 \\
-1.3\end{array}$ & $\begin{array}{l}+0.7 \\
+0.4 \\
+0.4 \\
+0.5\end{array}$ & $\begin{array}{l}+0.1 \\
-0.8 \\
-1.7 \\
-2.1\end{array}$ & $\begin{array}{l}-3.8 \\
-2.6 \\
-2.2 \\
-1.9\end{array}$ & $\begin{array}{l}-1.0 \\
-1.4 \\
-1.1 \\
-0.9\end{array}$ & $\begin{array}{l}-0.3 \\
-0.7 \\
-0.4 \\
-0.6\end{array}$ & $\begin{array}{l}-0.5 \\
-0.9 \\
-0.6 \\
-0.8\end{array}$ & $\begin{array}{l}-0.7 \\
-1.6 \\
-1.7 \\
-1.5\end{array}$ \\
\hline 2 & $\begin{array}{l}45-50 \\
50-55 \\
55-60 \\
60-65 \\
65-70 \\
70-75\end{array}$ & $\begin{array}{r}1 \\
3 \\
4 \\
7 \\
8 \\
11\end{array}$ & $\begin{array}{l}-1.6 \\
-1.9 \\
-2.2 \\
-1.9 \\
-2.0\end{array}$ & $\begin{array}{l}-1.8 \\
-2.2 \\
-1.5 \\
-1.8 \\
-2.1\end{array}$ & $\begin{array}{l}+2.1 \\
+1.9 \\
+2.0 \\
+1.3 \\
+1.8\end{array}$ & $\begin{array}{l}-0.7 \\
-0.3 \\
-1.2 \\
-0.6 \\
-0.1\end{array}$ & $\begin{array}{l}-2.0 \\
-1.8 \\
-1.5 \\
-1.0 \\
-1.0\end{array}$ & $\begin{array}{l}-1.0 \\
-1.5 \\
-1.7 \\
-1.3 \\
-1.2\end{array}$ & $\begin{array}{l}+1.3 \\
+0.9 \\
+1.8 \\
+0.9 \\
+1.4\end{array}$ & $\begin{array}{l}-0.7 \\
-0.6 \\
-0.9 \\
-1.2 \\
-0.6\end{array}$ & $\begin{array}{l}+0.2 \\
-0.7 \\
-0.6 \\
-0.5 \\
-0.1\end{array}$ \\
\hline 3 & $\begin{array}{l}45-55 \\
55-60 \\
65-75\end{array}$ & $\begin{array}{l}5 \\
5 \\
5\end{array}$ & $\begin{array}{l}-1.2 \\
-1.8 \\
-1.7\end{array}$ & $\begin{array}{l}-0.9 \\
-0.7 \\
-0.7\end{array}$ & $\begin{array}{l}+0.8 \\
+0.4 \\
+0.7\end{array}$ & $\begin{array}{l}+1.0 \\
+1.0 \\
-0.1\end{array}$ & $\begin{array}{l}-0.6 \\
-0.4 \\
-0.3\end{array}$ & $\begin{array}{l}+1.1 \\
+1.2 \\
+0.7\end{array}$ & $\begin{array}{l}+0.5 \\
+0.6 \\
+0.4\end{array}$ & $\begin{array}{l}+1.7 \\
+1.5 \\
+1.9\end{array}$ & $\begin{array}{l}+0.6 \\
+0.4 \\
+0.4\end{array}$ \\
\hline 4 & $45-50$ & 4 & $-1 \cdot 6$ & $-1 \cdot 4$ & -1.7 & $+0 \cdot 1$ & $-1 \cdot 1$ & $-1 \cdot 2$ & +0.3 & -0.8 & $-1 \cdot 7$ \\
\hline 5 & $55-60$ & 3 & +0.1 & +0.3 & +0.3 & $-0 \cdot 1$ & $+0 \cdot 1$ & $+0 \cdot 1$ & $+0 \cdot 1$ & -0.2 & -0.3 \\
\hline
\end{tabular}

Units $=$ z-score, above $(+)$ or below $(-)$ the mean.

Table 5 Static and dynamic histomorphometric parameters in cortical bone

\begin{tabular}{|c|c|c|c|c|c|c|c|c|c|c|c|}
\hline Group & $\begin{array}{l}\text { Age } \\
\text { (years) }\end{array}$ & No. & C.Th & $C V$ & C.WTh & C.OS & N.Oc.c & ES.s & N.Oc.s & $E R$ & $E P$ \\
\hline 1 & $\begin{array}{l}45-50 \\
50-55 \\
55-60 \\
60-65\end{array}$ & $\begin{array}{r}21 \\
33 \\
17 \\
4\end{array}$ & $\begin{array}{l}-0.5 \\
-0.4 \\
-0.4 \\
-0.4\end{array}$ & $\begin{array}{l}-0.6 \\
-0.7 \\
-1.0 \\
-0.5\end{array}$ & $\begin{array}{l}-1.4 \\
-3.1 \\
-2.9 \\
-2.1\end{array}$ & $\begin{array}{r}0.0 \\
-0.3 \\
-0.2 \\
-0.1\end{array}$ & $\begin{array}{l}+0.6 \\
+0.3 \\
+0.2 \\
+0.3\end{array}$ & $\begin{array}{l}+2.1 \\
+1.8 \\
+1.4 \\
+1.5\end{array}$ & $\begin{array}{l}+2.9 \\
+1 \cdot 2 \\
+1 \cdot 1 \\
+1 \cdot 2\end{array}$ & $\begin{array}{l}+1.3 \\
+1.4 \\
+0.8 \\
+0.7\end{array}$ & $\begin{array}{l}+0.2 \\
+0.3 \\
-0.1 \\
+0.8\end{array}$ \\
\hline 2 & $\begin{array}{l}45-50 \\
50-55 \\
55-60 \\
60-65 \\
65-70 \\
70-75\end{array}$ & $\begin{array}{r}3 \\
4 \\
7 \\
8 \\
11\end{array}$ & $\begin{array}{l}-1.5 \\
-1 \cdot 8 \\
-2.4 \\
-2.7 \\
-2.3\end{array}$ & $\begin{array}{l}-2.0 \\
-2.7 \\
-3.1 \\
-3.0 \\
-2.9\end{array}$ & $\begin{array}{l}-2 \cdot 0 \\
-2 \cdot 2 \\
-2 \cdot 1 \\
-1 \cdot 9 \\
-2 \cdot 0\end{array}$ & $\begin{array}{l}-0.7 \\
-1.1 \\
-1.0 \\
-1.0 \\
-0.8\end{array}$ & $\begin{array}{l}+0.9 \\
+13 \\
+0.8 \\
+0.9 \\
+1.0\end{array}$ & $\begin{array}{l}+1.6 \\
+2.0 \\
+1.5 \\
+1.7 \\
+1.8\end{array}$ & $\begin{array}{l}+2.4 \\
+1.9 \\
+1.9 \\
+1.9 \\
+1.6\end{array}$ & $\begin{array}{r}+0.7 \\
+0.6 \\
+0.5 \\
+0.1 \\
1.0\end{array}$ & $\begin{array}{l}-0.3 \\
+0.7 \\
-0.4 \\
-0.8 \\
+0.6\end{array}$ \\
\hline 3 & $\begin{array}{l}45-55 \\
55-60 \\
65-75\end{array}$ & $\begin{array}{l}5 \\
5 \\
5\end{array}$ & $\begin{array}{l}-0.4 \\
-0.8 \\
-0.9\end{array}$ & $\begin{array}{l}-1.2 \\
-1.7 \\
-1.5\end{array}$ & $\begin{array}{l}-0.4 \\
-0.6 \\
-0.5\end{array}$ & $\begin{array}{l}+0.2 \\
+0.5 \\
+0.5\end{array}$ & $\begin{array}{l}+1.0 \\
+1.2 \\
+1.2\end{array}$ & $\begin{array}{l}+1.2 \\
+1.6 \\
+1.6\end{array}$ & $\begin{array}{l}+1 \cdot 2 \\
+1 \cdot 7 \\
+1 \cdot 7\end{array}$ & $\begin{array}{l}+0.3 \\
+0.2 \\
+0.4\end{array}$ & $\begin{array}{l}+0.6 \\
+0.1 \\
+0.1\end{array}$ \\
\hline 4 & $45-50$ & 4 & $+0 \cdot 1$ & -0.6 & -2.9 & $-2 \cdot 2$ & -0.4 & $-1 \cdot 3$ & $-3 \cdot 7$ & $-1 \cdot 6$ & $-1 \cdot 7$ \\
\hline 5 & $55-60$ & 3 & 0.0 & $-0 \cdot 2$ & -0.2 & -0.3 & -0.1 & - & - & - & - \\
\hline
\end{tabular}

Units $=\mathrm{z}$-score, above $(+)$ or below $(-)$ the mean.

Group 3 comprised 16 (11\%) patients who had evidence of increased osteoid surface in their trabecular bone which was associated with a moderately reduced appositional rate. The osteoclast cell number and activity were increased in both the cortical and trabecular bone and, as such, the overall picture was similar to that of the common form of primary hyperparathyroidism seen in middle-aged women. ${ }^{24} 25$ Although the activation frequency in the trabecular bone was normal, the erosion rate was moderately increased. These patients all had normal serum parathyroid hormone, serum calcium, phosphate, and alkaline phosphatase concentrations.

Group 4 comprised seven (5\%) patients and was characterised by a noticeable reduction in both osteoblast and osteoclast number and activity in trabecular bone. This was associated with a moderately reduced rate of activation. Cortical wall thickness and the amount of cortical osteoid were reduced and were associated with an equally profound reduction in osteoclasis, particularly in the subcortical region. The eroded surfaces, rate of erosion, and the erosion period in the cortex were moderately reduced but cortical volume and cortical thickness were normal.

Group 5 comprised six (4\%) patients who were normal on histomorphometry despite radiological evidence of osteoporosis.

\section{Discussion}

A semiautomated histomorphometric technique was used to examine iliac crest bone samples from a large cohort of patients (aged from 45 to 75 years) with established postmenopausal osteoporosis. These patients were divided into five distinct subgroups based on histomorphometric data.

Histomorphometric subgroups, based mainly on the differential rate of bone turnover, have been described previously. ${ }^{92627}$ Most histomorphometric parameters have been described separately in previous studies but we believe that much of the debate on the type of histomorphometric heterogeneity seen in postmenopausal osteoporosis results from failure to recognise the full spectrum of changes that can occur in one group of patients, an aspect highlighted by this study. The most important findings of this study are the wide variation in the number and activity of bone surface osteoblasts and the lack of any correlation between osteoblastic and osteoclastic activity. Variation in these parameters can be illustrated by examining each individual group in more detail. The five subgroups can be generally defined as follows: group 1, decreased osteoblastic activity and normal osteoclasis in trabecular bone and decreased osteoblastic activity and normal or focally increased osteoclasis in cortical bone; 
group 2, decreased osteoblastic activity and increased osteoclasis in both trabecular and cortical bone; group 3, marginally increased osteoblastic activity and increased osteoclasis in both trabecular and cortical bone; group 4, decreased osteoblastic and osteoclastic activity in both trabecular and cortical bone; and group 5, no apparent cell abnormality.

The histomorphometric changes in groups 1 and 2 correspond to those described in the two main clinical subclasses of osteoporosis, namely postmenopausal (type 1) and senile (type 2) osteoporosis, ${ }^{1-4}$ the major difference being that none of our patients fulfilled the clinical criteria for senile osteoporosis. This illustrates that the changes previously described as characteristic of senile osteoporosis are present in one subgroup of patients with postmenopausal osteoporosis. Osteoblast activity was reduced in both groups 1 and $2^{3467}$ but osteoclastic bone resorption was increased in group 2. Although not identical, group 5 in this study corresponds in some respects to the patients described by Recker and Kimmel ${ }^{11}$ and group 3 is similar to those patients described by Melsen et $a l^{24}$ and Christiansen et al. ${ }^{25}$

This is, of course, a very general approach to analysis of the data. In cellular terms osteoblastic "activity" is a combination of a number of parameters (cell number, individual cell function, etc.) which can be looked at more closely based on the data collected. For instance, if the bone changes in group 1 are examined more closely, the histomorphometric data on the 76 younger postmenopausal women in this group show that the number of osteoblasts is reduced together with their ability to synthesise matrix. There is also a reduction in the amount of trabecular bone with an associated loss of trabecular integrity, suggesting that a local increase in bone destruction occurred leading to penetration of the trabecular plates. Despite fragmentation of trabeculae, which occurred in $82 \%$ of patients, there was no evidence of an increase in osteoclastic activity, suggesting that the pattern of cellular imbalance was different originally and that the pattern of cell changes that ultimately lead to clinically established osteoporosis may vary with time. ${ }^{10}$

Histomorphometric data are important for the management of patients with postmenopausal osteoporosis. In group 1 patients osteoblast number and activity are reduced. This is associated with a normal number of poorly functioning osteoclasts. Ideally, therefore, therapy should be directed towards increasing osteoblast recruitment and activity, whilst initially trying to prevent a concomitant increase in osteoclasis.

A similar exercise can be applied to the other groups. The extent of osteoblast dysfunction in group 2 is similar to that in group 1 ; however, group 2 patients have an increased number of osteoclasts. Although, as in group 1, the activity of individual osteoclasts was suppressed, osteoclasts were able to erode an increased proportion of trabecular surfaces, by virtue of their large numbers. The activation frequency was marginally reduced. Cortical bone parameters in group 2 patients exhibit similar changes to those in trabecular bone.

The most important consequence of these observations is that, at presentation, every patient can be regarded as having a specific cellular abnormality. Ideally, each patient should then receive a tailored treatment regimen. For instance, patients in group 1 are likely to respond positively to therapeutic regimens which promote bone formation, while those in group 2 should respond more favourably if potent antiresorptive agents are included.

These data should also be considered from two other perspectives. Firstly, they are expressed relative to age and sex matched "controls". Should fracture risk be an important end point in osteoporosis, all data (including age and sex matched normals) should be given relative to peak bone mass and its associated parameters. Were this the case, the negative $z$-scores in the osteoporotic patients would be even larger. Age and sex matched controls have been used here to stress how women with postmenopausal osteoporosis differ from women of the same age without the syndrome because it could be argued that treatment might be aimed at "normalising" a defect rather than at trying to reattain the ideal, as represented by the peak parameters.

Secondly, the approach adopted here for the analysis of osteoporosis is quite different to that used by other authors. There is a longstanding discussion in the literature of the validity of data analysis in patients with type 1 osteoporosis. ${ }^{28}$ These researchers classified their patients clinically (mainly based on age and clinical characteristics) and then attempted to identify a unified histomorphometric defect to explain bone loss. We believe that the approach adopted in this study is of greater clinical and therapeutic value.

1 Riis BJ, Rodbro O, Christiensen C. The role of serum concentration of sex steroids and bone turnover in the development and occurrence of postmenopausal osteoporosis Calcific Tissue Int 1986;38:318-22.

2 Eriksen EF, Hodgson SF, Eastell R, Cedal SL, O'Fallon WM, Riggs BL. Cancellous bone remodelling in type I (postmenopausal) osteoporosis: quantitative assessmen of rates of formation, resorption, and bone loss at tissue and cellular levels. F Bone Miner Res 1990;5:311-19.

3 Parfitt AM. Bone remodelling in the pathogenesis of osteoporosis. Medical Times 1981;109:80-92.

4 Parfitt AM. Age-related structural changes in trabecular and cortical bone: cellular mechanism and biochemical consequences. a) differences between rapid and slow consequences. a) differences between rapid and slow
bone loss. b) localised bone gain. Calcific Tissue Int bone loss. b) localised bon

5 Heaney RP, Recker RR, Saville PD. Menopausal changes in bone remodelling. $\mathcal{F}$ Lab Clin Med 1978;92:964-70.

6 Kleerekoper M, Villaneuvar AR, Stanciu J, Rao DS, Parfitt AM. The role of three dimensional trabecular microstructure in pathogenesis of vertebral compression fractures. Calcif Tissue Int 1985;37:594-7.

7 Vesterby A, Gundersen HJG, Melsen F. Star volume of marrow space and trabeculae of first lumbar vertebra: Sampling efficiency and biological variation. Bone 1989; 10:7-13.

8 Parfitt AM. Bone remodelling: relationship to the amount and structure of bone, and pathogenesis and prevention of fractures. In: Riggs BL, Melton LJ, eds. Osteoporosis, etiology, diagnosis and management. New York: Raven Press, 1988:45-93.

9 Whyte MP, Bergfeld MA, Murphy WA, Avioli LV, 
Teitelbaum SL. Postmenopausal osteoporosis. A heterogenous disorder as assessed by histomorphometric analysis of iliac crest bone from untreated patients. $A m \mathcal{F}$ Med 1982;72:193-202.

10 Meunier PJ, Sellami S, Briacorn D, Edouard C. Histological heterogeneity of apparently idiopathic osteoporosis. In: DeLuca HF, Frost HM, Jee WSS, Johnston CC, Parfitt AM, eds. Osteoporosis. Recent advances in pathogenesis and treatment. Baltimore, MD: University Park Press, 1981:293-301.

11 Recker RR, Kimmel DB. Changes in trabecular microstructure in osteoporosis occur with normal bon remodelling dynamics. I Bone Miner Res 1991;6 (Suppl):S225.

12 Wand JS, Green JR, Hesp R, Bradbeer JN, Sambrook PM, Smith $\mathrm{T}$, et al. Bone remodelling does not decline after menopause in vertebral fracture osteoporosis. Bone Minop 1992;17:361-75.

13 Keshawarz NMS, Recker RR. Expansion of medullary cavity at the expanse of cortex in postmenopausal osteoporosis. Metab Bone Dis Relat Res 1984;5:223-8.

14 Brown JP, Delmas PD, Arlot AM, Meunier PJ. Active bone turnover of the cortico-endosteal envelope in postmenopausal osteoporosis. 7 Clin Endocrinol Metab 1987 64:954-9.

15 Riggs BL, Melton LJ III. Evidence for two distinct types of osteoporosis. Am F Med 1983;75:899-901.

16 Melsen F, Mosekilde L. The role of bone biopsy in diagnosis of metabolic bone disease. Orthop Clin North Am nosis of metabolic

17 Parfitt AM, Mathews CHE, Villaneuva AR, Kleerekoper M, Frame B, Rao DS. Relationship between surface volume and thickness of iliac trabecular bone in aging and osteoporosis. f Clin Invest 1983;72:1396-409.

18 DeLesse A. Procédé mécanique pour déterminer la composition des roches. Ann Mines 1884;13:339.
19 Frost HM. Histomorphometry: techniques and interpretation. In: RR Recker, ed. Boca Raton, FL: CRS Press, 1983:109-32.

20 Eriksen EF, Hodgson SF, Eastell R, Cedal SL, O'Fallon WM, Riggs BL. Cancellous bone remodelling in type I (postmenopausal) osteoporosis: quantitative assessmen of rates of formation, resorption, and bone loss at tissue and cellular levels. $\mathscr{F}$ Bone Miner Res 1990;5:31 1-19.

21 Compston JE, Mellish RWE, Croucher P, Newcombe R Garrahan NJ. Structural mechanisms of trabecular bone loss in man. Bone Miner 1989;6:339-50.

22 Parfitt AM, Drezner MK, Glorieux FH, Kanis JA Maluche H, Meunier PJ, et al. Bone histomorphometry: Standardisation of nomenclature, symbol, and units. f Bone Min Res 1987;2:595-610.

23 Rehman MTA, Hoyland JA, Denton J, Freemont AJ. Age related histomorphometric changes in bone in normal British men and women. $f$ Clin Pathol 1994;47:529-34.

24 Melson F, Mosekilde L. The role of bone biopsy in diagnosis of metabolic bone disease. Orthop Clin North Am 1981;12:571-602.

25 Christiansen P, Steniech T, Brocketedt H, Mosekilde L Hessove I, Melsen F. Primary Hyperparathyroidism: Iliac crest cortical thickness, structure and remodelling evaluated by histomorphometric methods. Bone 1993; 14:755-62.

26 Arlot ME, Delmas PD, Faculte A, Chappard D, Meunier PJ. Impaired osteoblast function in osteoporosis: comparison between calcium balance and dynamic histomorphometry. Osteoporosis Int 1990;1:41-9.

27 Kimmel DB, Recker RR Gallaghar JC, Vaswani AS, Aloia JF. A comparison of iliac crest bone histomorphometric JF. A comparison of iliac crest bone histomorphometric data in postmenopausal ost

28 Parfitt AM. Bone remodelling in type I osteoporosis [letter]. F Bone Miner Res 1991;6:95-6. 